\title{
A Prediction Framework for Cardiac Resynchronization Therapy Via 4D Cardiac Motion Analysis
}

\author{
Heng Huang ${ }^{1}$, Li Shen ${ }^{3}$, Rong Zhang ${ }^{1}$, Fillia Makedon ${ }^{1}$, \\ Bruce Hettleman $^{2}$, and Justin Pearlman ${ }^{1,2}$ \\ 1 Department of Computer Science, Dartmouth College, Hanover, NH 03755 \\ 2 Department of Cardiology, Dartmouth Medical School, Lebanon, NH 03756 \\ ${ }^{3}$ Computer and Information Science Department, Univ. of Mass. Dartmouth, MA 02747 \\ hh@cs. dartmouth. edu
}

\begin{abstract}
We propose a novel framework to predict pacing sites in the left ventricle (LV) of a heart and its result can be used to assist pacemaker implantation and programming in cardiac resynchronization therapy (CRT), a widely adopted therapy for heart failure patients. In a traditional CRT device deployment, pacing sites are selected without quantitative prediction. That runs the risk of suboptimal benefits. In this work, the spherical harmonic (SPHARM) description is employed to model the ventricular surfaces and a novel SPHARM-based surface correspondence approach is proposed to capture the ventricular wall motion. A hierarchical agglomerative clustering technique is applied to the time series of regional wall thickness to identify candidate pacing sites. Using clinical MRI data in our experiments, we demonstrate that the proposed framework can not only effectively identify suitable pacing sites, but also distinguish patients from normal subjects perfectly to help medical diagnosis and prognosis.
\end{abstract}

\section{Introduction}

Heart failure, also called congestive heart failure, is a major health problem that continues to increase in prevalence. It is a disorder in which the heart loses its ability to pump blood efficiently. Low cardiac output resulting from heart failure may cause the body's organ systems to fail. As one important part of the problems, the walls of the left ventricle (LV) are unable to contract synchronously.

Over the past decade, investigators [2] have established the feasibility of placing multiple pacing leads of pacemaker to improve the activation synchrony (sameness of activation time) of LV and biventricle. Based on these studies, a promising therapeutic option, called cardiac resynchronization therapy (CRT), has been proposed as an alternative treatment in patients with severe, drug-refractory heart failure. It is aimed at correcting contraction delays that result in different regions of the heart not working optimally in concert [1].

Although clinical trials have confirmed that CRT improved clinical symptoms, increased exercise capacity, and led to cessation or even reversal of chronic chamber remodeling, a significant minority seem not to benefit. [3] There are a lot of potential explanations for the CRT failure cases, and improper surgical placement of the LV lead is one of the most important reasons. Improvements in the methods of identification of likely responsive implantation placements are needed. 
The initial CRT device utilization incorporates right ventricle and LV pacing sites, but a right ventricle pacing site is not required for hemodynamic benefit in many patients [4]. Since LV pacing alone has the almost similar benefit with biventricular pacing, it is used more popular in the CRT system. Thus, in this paper, we focus on the CRT with LV pacing sites.

The principal goal of the present study is to efficiently predict the optimal LV pacing sites that should be stimulated by electrical impulses of pacemaker and provide the corresponding parameters (timing delay, etc.) to help the programmable device. In order to identify the optimal pacing sites, the mechanical dyssynchrony is directly analyzed by the spatio-temporal modeling. Based on the fact that ventricular wall thickening and motion reflect activation, we build an integrated framework to estimate the most effective places for implanting the pacemaker to achieve maximized CRT benefit. Given a stack of cardiac MRI, both endocardium and epicardium are reconstructed, and the optimal correspondences between them are established by minimizing the surface Euclidean distance. LV wall motion is described by the three dimensional (3D) wall thickness change that is computed using the reconstructed LV surfaces. After applying the hierarchical clustering method on a time series of wall thickness measurements, we can find candidate pacing sites with abnormal local motion. Our experiments also show that this study can be used to distinguish patients and normal subjects and judge the disease degree.

\section{Methods}

\subsection{Spatio-Temporal LV Motion Modeling}

In order to quantify the ventricular mechanical asynchrony or synchrony that can directly help determine optimal treatment, we develop our spatio-temporal model to describe a temporal sequence of wall thickness changing during a heart cycle.

Surface reconstruction. We reconstruct both endocardium and epicardium of the LV by using the spherical harmonic (SPHARM) method, which was introduced by Brechbühler, Gerig and Kübler [5] for modeling any simply connected 3D object. The object surface is parameterized as $\mathbf{v}(\theta, \phi)=(x(\theta, \phi), y(\theta, \phi), z(\theta, \phi))^{T}$ using a pair of spherical coordinates $(\theta, \phi)$, where the parameterization aims to preserve the area and minimize the angle distortion. Thus, $\mathbf{v}(\theta, \phi)$ becomes a vector of three spherical functions that can be expanded using spherical harmonics $Y_{l}^{m}(\theta, \phi)$ as follows,

$$
\mathbf{v}(\theta, \phi)=\sum_{l=0}^{\infty} \sum_{m=-l}^{l} \mathbf{c}_{l}^{m} Y_{l}^{m}(\theta, \phi),
$$

where $\mathbf{c}_{l}^{m}=\left(c_{l x}^{m}, c_{l y}^{m}, c_{l z}^{m}\right)^{T}$ are expansion coefficients that can be used to reconstruct the object surface. SPHARM has been used by Gerig and Styner in many medical imaging applications (e.g., shape analysis of brain structures [6, 7, 8]). It has also been used for shape modeling and functional analysis of cardiac MRI [9]. Since SPHARM provides an implicit correspondence between surfaces of $3 \mathrm{D}$ objects, it is suitable to be used to analyze the LV wall motion during heart cycle. 
In our cardiac MRI data sets, each MRI sequence holds seventeen temporal phases per heartbeat. Since the LV deformation is exhibited by the thickness change of the wall between endocardium and epicardium, we use 17 SPHARM reconstructed surface pairs (including both endocardium and epicardium) to describe the LV contraction and dilation during a whole heart cycle.

Surface correspondence. In order to measure the wall thickness at each surface location as well as compare thickness changes between different time points, a registration step is necessary for aligning all the reconstructed epicardial surfaces together. Given two SPHARM models, we establish their surface correspondence by minimizing the Euclidean distances between their corresponding surface locations. Formally, for two surfaces given by $\mathbf{v}_{1}(s)$ and $\mathbf{v}_{2}(s)$, their distance $D\left(\mathbf{v}_{1}, \mathbf{v}_{2}\right)$ is defined as [7]

$$
D\left(\mathbf{v}_{1}, \mathbf{v}_{2}\right)=\left(\oint\left\|\mathbf{v}_{1}(s)-\mathbf{v}_{2}(s)\right\|^{2} d s\right)^{1 / 2}=\left(\sum_{f \in\{x, y, z\}} \sum_{l=0}^{L} \sum_{m=-l}^{l}\left(c_{l f_{1}}^{m}-c_{l f_{2}}^{m}\right)^{2}\right)^{1 / 2} .
$$

The epicardial surface in the first time phase (diastolic phase in our MRI data) during heartbeat is used as the template. For any other epicardial surface in the same sequence, we align it to the template by rotating its parameter net [10] so that the surface distances $D\left(\mathbf{v}_{1}, \mathbf{v}_{i}\right),(i=2, \ldots, 17)$ between them is minimized. Given an aligned surface sequence, we use the same method to align the endocardium to the epicardium in the same timing phase.

Wall motion series. Because the wall thickness change of LV directly shows the wall motion in $3 \mathrm{D}$ space during a heart cycle, we can use it as the wall motion descriptor. In this study, we observe that the distance between the corresponding points (i.e., with the same $(\theta, \phi))$ on the endocarium and epicardium surfaces, can be directly used to approximate the wall thickness, since we have already minimized this distance in the previous step. In addition, the underlying equal area parameterization implies the correspondence relationships between any pair points on these two surfaces are reasonable and effective.

After that, we create the wall motion series that includes the thickness values for each corresponding surface location at each time phase during a heart cycle, from enddiastolic phase to next end-diastolic one. Since we are only interested in the LV wall motion, we ignore the points appearing on the top of reconstructed surfaces. Even if only one point whose thickness value in the wall motion series appears on the top of its surface, the whole motion series is discarded.

\subsection{Clustering Based Pacing Sites Search}

After the above steps, a set of motion series are used to present the LV wall contraction. Given a pair of $(\theta, \phi)$, we use $\boldsymbol{w}(\theta, \phi)=\left\{w_{1}(\theta, \phi), w_{2}(\theta, \phi), \ldots, w_{n}(\theta, \phi)\right\}$ to denote its corresponding wall motion series, where $w_{i}(\theta, \phi)$ is the wall thickness value of wall motion phase $i$ corresponding to the parametrized point $(\theta, \phi)$ on the epicrdium.

Similarity measurement. Formlly, given two wall motion series $\boldsymbol{w}\left(\theta_{x}, \phi_{x}\right)$ and $\boldsymbol{w}\left(\theta_{y}, \phi_{y}\right)$, we employ the following formulae to measure the distance or dissimilarity between them: 


$$
\begin{gathered}
d_{\text {corr }}\left(\boldsymbol{w}\left(\theta_{x}, \phi_{x}\right), \boldsymbol{w}\left(\theta_{y}, \phi_{y}\right)\right)=1-r\left(\boldsymbol{w}\left(\theta_{x}, \phi_{x}\right), \boldsymbol{w}\left(\theta_{y}, \phi_{y}\right)\right)= \\
1-\left[\sum_{i=1}^{n}\left(\frac{w_{i}\left(\theta_{x}, \phi_{x}\right)-w_{\text {mean }}\left(\theta_{x}, \phi_{x}\right)}{\sigma_{x}}\right)\left(\frac{w_{i}\left(\theta_{y}, \phi_{y}\right)-w_{\text {mean }}\left(\theta_{y}, \phi_{y}\right)}{\sigma_{y}}\right)\right] / n
\end{gathered}
$$

where $\sigma=\sqrt{\left(\sum_{i=1}^{n}\left(w_{i}(\theta, \phi)-w_{\text {mean }}(\theta, \phi)\right)^{2}\right) / n} \cdot r\left(\boldsymbol{w}\left(\theta_{x}, \phi_{x}\right), \boldsymbol{w}\left(\theta_{y}, \phi_{y}\right)\right)$ is the Pearson correlation coefficient of two wall motion series, $w_{\text {mean }}(\theta, \phi)$ is the mean of wall motion series, and $\sigma$ is the standard deviation of $\boldsymbol{w}(\theta, \phi)$. The Pearson correlation coefficient is always between -1 and 1 , and we normalize distance function as $d_{\text {corr }} / 2$ (the result will change from 0 to 1 ) in our experiments.

Hierarchical clustering. By combining or clustering similar wall motion series we can identify groups of wall motion series that are the main trend of LV contraction and dilation for different locations in the $3 \mathrm{D}$ space. To group similar wall motion series together, we employ hierarchical agglomerative clustering approach [11], which is a bottom-up clustering method where clusters can have sub-clusters.

The hierarchical clustering result, a dendrogram, is a binary tree (see Fig. 1 for an example) in which each data point corresponds to a leaf nodes, and distance from the root to a subtree indicates the similarity of subtrees-highly similar nodes or subtrees have joining points that are farther from the root.

Sweep line method. We move the horizontal sweep-line from top to bottom in the dendrogram result (for example, the "sweep-line 1" in Fig. 1) to get the abnormal clusters (small clusters) that have a large dissimilarity to the main cluster. Note that the pacemaker system uses electrical impulses to adjust the sites whose contraction characteristics are considerably different from other sites'. Thus, hierarchical clustering results can help us to find these location candidates for installing the pacing leads.

\subsection{Pacing Site Pre-filtering}

Cross correlation. In order to set the electrical impulses in a pacemaker system, a technician still needs to know the timing delay value between the pacing position and a common position. Hence we use cross correlation method to acquire such a value between the wall motion series. For two wall motion series $\boldsymbol{w}\left(\theta_{x}, \phi_{x}\right)$ and $\boldsymbol{w}\left(\theta_{y}, \phi_{y}\right)$, the correlation function of two wall motion series is defined as:

$$
c c_{x y}(t)=\boldsymbol{w}\left(\theta_{x}, \phi_{x}\right) \star \boldsymbol{w}\left(\theta_{y}, \phi_{y}\right)=\sum_{m=1}^{n} w_{m}\left(\theta_{x}, \phi_{x}\right) w_{m+t}\left(\theta_{y}, \phi_{y}\right)
$$

where " $\star$ " is the correlation operator, and $t=0,1, \ldots, n-1$. If $t=t_{0}$ satisfies $c c_{x y}\left(t_{0}\right)=\max \left(c c_{x y}(t)\right)$ for $t \in[0, n-1]$, then the wall motion series $\boldsymbol{w}\left(\theta_{x}, \phi_{x}\right)$ shifts $t_{0}$ to get the maximum correlation with the wall motion series $\boldsymbol{w}\left(\theta_{y}, \phi_{y}\right)$. Thus, $t_{0}$ is the timing shift (or delay). The timing period between two neighbouring phases can be calculated using the heartbeat velocity. Thus, the timing delay can be calculated by: timing delay $=t_{0} \times($ a heartbeat period/the number of phases $)$. 
Pacing sites selection. We introduce a filtering step on the pacing site candidates list, because a few of them don't have contraction timing delay to the normal activation. After picking up the site candidates, there is a single big cluster in the dendrogram, called the main cluster (see Fig. 1 for a marked sample main cluster). The wall motion series (average motion series) of the main cluster is regarded as the normal wall motion variation of the LV, for example the square-line in Fig. 2 and Fig. 3. Using the contraction timing delay between pacing site candidates and main cluster, we filter out the site candidates without contraction delay.

In the implantation, a doctor still needs to test the lead to see whether a candidate location is suitable for pacing, because the pacing lead cannot be placed into some regions of left ventricle (such condition normally is created by epicardial scar or unacceptable phrenic nerve stimulation, etc). Based on the dendrogram result, we provide the location candidates for implanting and they are rated by the distances from the main cluster, which is described below.

\section{Results and Discussions}

We have implemented our pacing site prediction framework using Matlab 6.5. To show the effectiveness of this framework, we use cardiac MRI data from 20 patients in our experiments, where half of them have heart failure problems. These experiments are conducted on a PC with a $2.40 \mathrm{GHz} \mathrm{CPU}$ and $512 \mathrm{MB}$ main memory. Note that the patients are diagnosed by specialized physicians, and these diagnostic results are used to validate our results in the experiments.

For convenience, we allocate a number to each wall motion series. From apex to basis of the $\mathrm{LV}, 1 \sim 96$ are used to mark the points of wall motion series level by level. Therefore, the points represented by consecutive numbers are in the neighbour locations on the surface, and the points with small numbers should be close to apex and the points with large numbers should be close to the basis of the LV.

Fig. 1 shows the hierarchical clustering result of a patient with heart failure problem. The dendrogram consists of a main cluster and several other small ones. The locations corresponding to the motion series in those small clusters are selected as the candidate pacing sites. Note that a single small cluster may include multiple regions on the LV, since the different regions may have similar motion behaviors. In Fig. 1, $\{92,93\}$ (close to the basis of LV) is the top-priority option for resynchronization therapy, and the next pacing candidates that should be considered are $\{77,78,79\}$ and $\{30,31\}$.

Since the distance function used by us cannot discriminate the timing delay between wall motion series, pre-filtering step should be executed here. Fig. 2 and Fig. 3 show the pacing sites filtering step. In Fig. 2, the curve with square tags is the average motion series of the main cluster in Fig. 1 and the curve with circle tags is the average motion series of region $\{92,93\}$. Because there is no timing delay between the main cluster and this region, it is filtered out, although their average wall motion series is very different from the main cluster's. Regions $\{77,78,79\}$ and $\{30,31\}$ still remain in the candidate list, since obvious timing delays are observed in Fig. 3. After filtering step, our results can be used for pacemaker implantation. As we mentioned before, the pacing lead cannot be placed into some particular regions of the LV. The physician will test the pacing 


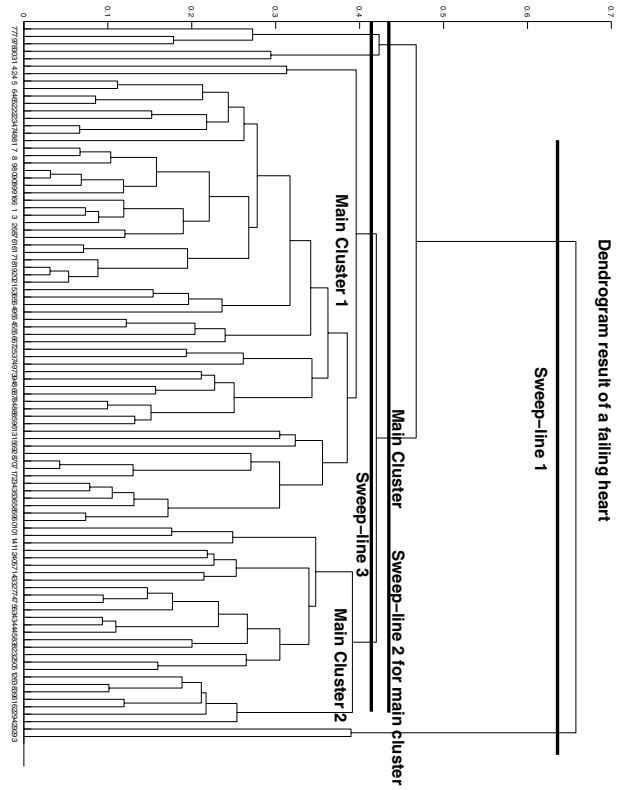

Fig. 1. Dendrogram result of a failing heart. The $x$-axis label represents the number of wall motion series. The $y$-axis label corresponds to the distance between clusters. The dendrogram is cut into clusters by the "sweepline 3".

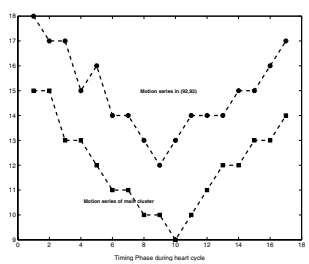

Fig. 2. There is no obvious timing delay between the average wall motion series of the main cluster (square-curve) and the motion series of region $\{92,93\}$ (circle-curve)

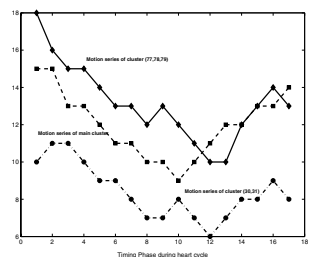

Fig. 3. There is a contraction timing delay between the main cluster and regions $\{77,78,79\}$ (diamond-curve) and $\{30,31\}$ (circle-curve)

lead on candidate pacing sites according to the suggested site ordering until they find a suitable region for fixing the tip of pacing lead. If the list is empty and a suitable site isn't found, we will continue to select a lower value sweep-line in the dendrogram result, for example, the "sweep-line 2" and "sweep-line 3" in Fig. 1. Because the candidates list includes locations with notable asynchronous contraction and timing delay, the optimal resynchronization therapy can be obtained after adding electrical pulse into these candidates. These sites are potentially good candidates to implant the pacemaker for a more efficient CRT. Furthermore, in some clinical cases, a physician may want to use multiple sites in left ventricular pacing for cardiac resynchronization, and they can select additional locations from the candidates list.

We test our methods on the MRI data of both normal and failing hearts. The dendrogram results of the normal hearts are very different from the failing ones. In the normal heart dendrogram, see Fig. 4 if the value of sweep-line is selected as $\geq 0.3$ (the distance between clusters), we obtain only one single main cluster without any other small clusters. This matches our intuition, since the wall motion of a normal heart tends to be synchronous and so the motion difference on different surface locations are very small. Thus our analysis may be useful in identifying patient candidates for helping diagnosis. After obtaining twenty dendrograms for all subjects, for each single case, we move the sweep-line from top to bottom until the result contains exactly two clusters. Fig. 5 


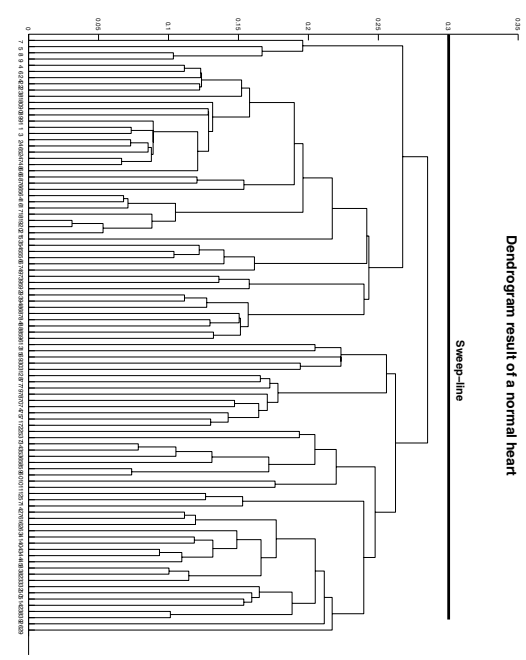

Fig. 4. Dendrogram result of a normal heart. The $x$-axis label represents the number of wall motion series. The $y$ axis label corresponds to the distance between clusters.

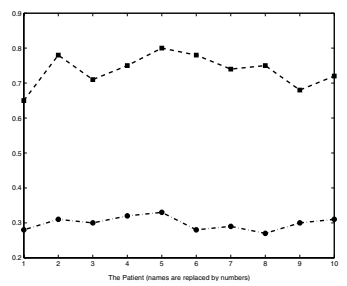

Fig. 5. The cutoff value of the sweep-line

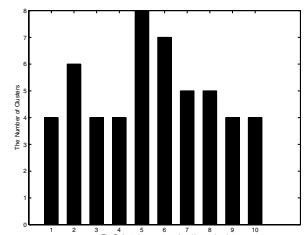

Fig. 6. The number of small clusters extracted from the abnormal subjects

summarizes the final values of these sweep-lines, sorted in two groups - one group holds 10 low values, and the other one holds 10 high values. The clinical diagnosis indicates that all low value points (circles) correspond to normal hearts and all high value ones (squares) correspond to failing hearts. Note that there is a big gap between these two lines, and so such hierarchical clustering results can actually separate subjects with heart failure from normal subjects. This observation is helpful for heart failure diagnosis and prognosis. The value $0.4 \sim 0.5$ seems to be a good threshold for the sweep-line to distinguish failing hearts from normal hearts in our data.

In the failing heart data set, we move the sweep-line to extract all small clusters (this sweep-line may separate the main cluster into two or more main clusters). "Sweepline 3" in Fig. 1 is such an example and in this case we have five clusters: $\{92,93\}$, $\{77,78,79\},\{30,31\}$, main cluster 1 and main cluster 2. Fig. 6 shows the number of clusters gotten from these 10 abnormal subjects, without counting the main cluster. In this result, the dendrogram result of patient $\# 5$ has more small clusters and the patient also has worse heart failure symptom. It is often the case that, the more severe heart failure the patient has, the more asynchronous wall contraction the LV performs. Our results reveal such a reasonable relationship between the number of pacing site candidates and the degree of patients' symptom.

\section{Conclusion}

In this paper, we propose a new pacing sites prediction framework that is based on spatio-temporal analysis of cardiac motion patterns and hierarchical clustering method. 
It can automatically generate the candidate site list to help a physician to localize the pacing areas. Blinded analysis of clinical MRI data demonstrates that our approach can not only identify pacing sites in the LV for assisting pacemaker implantation in CRT, but also be used to help medical diagnosis of heart failure. Since the bi-ventricular pacing is useful for some clinical cases, our further study will be carried out on the prediction of its pacing sites. This further study will be combined with our current prediction system to improve the accuracy and feasibility of estimated results.

\section{References}

1. Khaykin, Y., Saad, E.B., Wilkoff, B.L.: Pacing in heart failure: the benefit of resynchronization. Cleve. Clin. J. Med. 70(10) (2003) 841-865

2. Trautmann, S.I., Kloss, M., Auricchio, A.: Cardiac resynchronization therapy. Curr. Cardiol. Rep. 4(5) (2002) 371-378

3. Auricchio, A., Stellbrink, C., Sack, S., et al.: Long-term clinical effect of hemodynamically optimized cardiac resynchronization therapy in patients with heart failure and ventricular conduction delay. J. Am. Coll. Cardiol. 39(12) (2002) 2026-2033.

4. Stevenson, W.G., Sweeney, M.O.: Single Site LV Pacing for Cardiac Resynchronization. Circulation. 90(5) (2004) 483-484

5. Brechbühler, Ch., Gerig, G., and Kübler, O.: Parametrization of closed surfaces for 3D shape description. Computer Vision and Image Understanding. 61(2) (1995) 154-170

6. Styner, M., Gerig G.: Three-Dimensional Medial Shape Representation Incorporating Object Variability. Proc. IEEE Conf. Computer Vision and Pattern Recognition. (2002) 651-656

7. Gerig, G., and Styner, M.: Shape versus Size: Improved Understanding of the Morphology of Brain Structures. International Conf. on Medical Image Computing and Computer Assisted Intervention. LNCS 2208 (2001) 24-32

8. Styner, M., Lieberman, A.J., Pantazis, D., and Gerig, G.: Boundary and Medial Shape Analysis of the Hippocampus in Schizophrenia. Med. Im. Ana. MEDIA. 8(3) (2004) 197-203.

9. Huang, H., Shen, L., Ford, J., Makedon, F., Zhang, R., et al.: Functional analysis of cardiac MR images using SPHARM modeling. Proceedings of the SPIE 5747 (2005) 1384-1391.

10. Huang, H., Shen, L., Zhang, R., Makedon, F., Hettleman, B., Pearlman, J.: Surface Alignment of 3D Spherical Harmonic Models: Application to Cardiac MRI Analysis. International Conf. on Medical Image Computing and Computer Assisted Intervention. (2005)

11. Alpaydin, E.: Introduction to Machine Learning. The MIT Press, (2004) 